\title{
Cesium carbonate mediated exclusive dialkylation of active methylene compounds
}

\author{
Ulaganathan Sankar, Chandran Raju, Ramakrishnan Uma*
}

Department of Chemistry, Pachaiyappa's College, University of Madras, Chennai - 600030, India

\begin{tabular}{|c|c|}
\hline A R T I C LE I N F O & A B S T R A C T \\
\hline $\begin{array}{l}\text { Article history: } \\
\text { Received March } 30,2012 \\
\text { Received in Revised form } \\
\text { May } 21,2012 \\
\text { Accepted } 25 \text { May } 2012 \\
\text { Available online } \\
\text { 25 May } 2012 \\
\end{array}$ & $\begin{array}{l}\text { Active methylene compounds are regioselectively dialkylated by variety of alkyl halides using } \\
\text { cesium carbonate in quantitative yield. The reaction yielded exclusively dialkylated products } \\
\text { with no intermediate monoalkyaltion or mixture of products. }\end{array}$ \\
\hline $\begin{array}{l}\text { Keywords: } \\
\text { Balanced base } \\
\text { Aprotic solvent } \\
\text { Active methylene compounds } \\
\text { Reactive halides } \\
\text { Complete conversion } \\
\end{array}$ & (C) 2012 Growing Science Ltd. All rights reserved. \\
\hline
\end{tabular}

\section{Introduction}

In recent years, cesium carbonate has found extensive applications as an excellent base for a variety of synthetic transformations ${ }^{1-13}$ and has received even industrial acceptance. In most of the cases, it is superior to other bases in terms of yield, reaction time, reaction temperature and sensitivity towards moisture, thus compensating the rather high cost of this reagent compared to that of potassium carbonate or other cheaper bases. It is easy to handle using readily available commercial starting materials without requiring strict exclusion of moisture in the system. Its basic strength is shown by the fact that it is the base of choice for reactions that are too sensitive towards strong bases or reactions that require a "Balanced base," stronger than other carbonates and weaker than hydroxides or alkoxides. It is compatible with a variety of functional groups. It has widely been employed for selective mono alkylation of amines ${ }^{1}$, O-alkylation of phenols ${ }^{2}$, O-alkylation of carboxylic acids ${ }^{3}$ and S-alkylation ${ }^{4}$.

* Corresponding author. Tel.: +91-9444253900

E-mail addresses: saisaidas1232002@yahoo.co.in (R. Uma)

(C) 2011 Growing Science Ltd. All rights reserved.

doi: $10.5267 /$ j.ccl.2012.5.003 
Surprisingly, its use in C-C bond formation reactions has not been explored much, except for a few reports on the Michael addition ${ }^{5}$ and a solitary report on the alkylation of acetamidomalonicester ${ }^{6}$.

Alkylation of active methylene compounds are generally accomplished using bases like sodium hydride, sodium ethoxide, potassium tert-butoxide, potassium phosphate and potassium carbonate. In many of the cases, yields are only moderate and mixtures of mono, di and $O$-alkylated product as well as a small amount of condensation product are formed. Sometimes, separation and isolation of the pure product from this mixture poses problems. Therefore alternative approaches to these products need to be developed. In this letter we describe a highly efficient and practical Method for the dialkylation of active methylene compounds by using cesium carbonate in dimethylformamide.

\section{Results and Discussion}

Stirring a solution of acetyl acetone $(10 \mathrm{mmol})$ in DMF $(15 \mathrm{~mL})$ with allyl bromide $(25 \mathrm{mmol})$ and cesium carbonate $(20 \mathrm{mmol})$ at r.t., for 45 mins resulted in complete consumption of acetyl acetone and afforded the diallylated product in nearly quantitative yield (Table 1, entry 1). As shown in Table 1 , several active methylene compounds ( $\beta$-diketones, $\beta$-ketoesters and nitriles) can undergo alkylation smoothly with several reactive halides in the presence of cesium carbonate at room temperature itself, affording the corresponding di $C$-alkylated products in good to excellent yield and in good purity in a reasonably short period of time. In the case of diethyl malonate and benzonitrile, no reaction occurred at ambient temperature, but complete conversion was observed at $70{ }^{\circ} \mathrm{C}$ (Table 1, entries 1112). In all cases, crude product itself was sufficiently pure $(>95 \%)$, except in the case of alkylation with benzyl bromide (Table 1, entries 13-17).

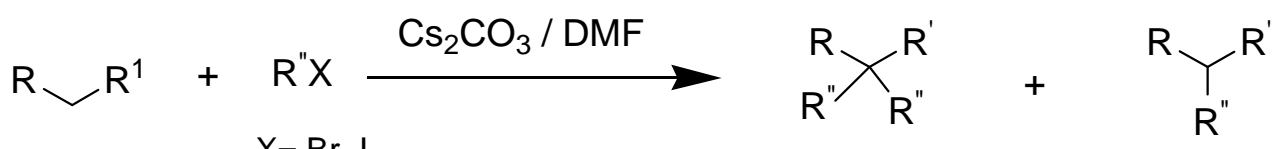

$$
\begin{aligned}
& \mathrm{X}=\mathrm{Br}, \mathrm{I} \\
& >99 \% \quad 0 \%
\end{aligned}
$$

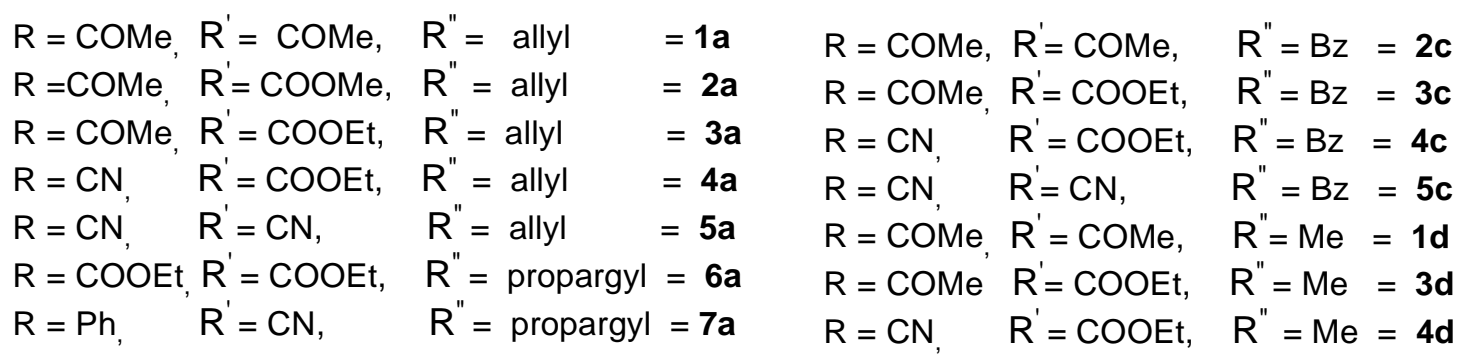

$$
\begin{aligned}
& \mathrm{R}=\mathrm{COMe}, \mathrm{R}^{\prime}=\text { COMe }, \quad \mathrm{R}^{\prime \prime}=\text { propargyl }=\mathbf{1 b} \\
& \mathrm{R}=\mathrm{COMe}, \mathrm{R}^{\prime}=\mathrm{COOMe}, \mathrm{R}^{\prime \prime}=\text { propargyl }=\mathbf{2} \mathbf{b} \\
& \mathrm{R}=\mathrm{COMe}, \mathrm{R}^{\prime}=\text { COOEt }, \quad \mathrm{R}^{\prime \prime}=\text { propargyl }=\mathbf{3} \mathbf{b} \\
& \mathrm{R}=\mathrm{CN}, \quad \mathrm{R}^{\prime}=\mathrm{COOEt}, \quad \mathrm{R}^{\prime \prime}=\text { propargyl }=\mathbf{4 b} \\
& \mathrm{R}=\mathrm{CN}, \quad \mathrm{R}^{\prime}=\mathrm{CN}, \quad \mathrm{R}^{\prime \prime}=\text { propargyl }=\mathbf{5 b}
\end{aligned}
$$

Scheme 1. Dialkylation of active methylene compounds.

In these cases benzyl bromide was used in slight excess, and hence the product was accompanied with unreacted excess benzyl bromide which was easily removed by crystallization of the crude product from n-hexane/ethyl acetate (9:1). 
Table 1. Alkylation of active methylene compounds

\begin{tabular}{|c|c|c|c|c|c|}
\hline Entry & Active methylene compounds & Alkyl halide (RX) & Condition & Product $^{\mathrm{a}, \mathrm{b}}$ & Yield (\%) \\
\hline 1 & & allyl bromide & r.t., $45 \mathrm{~min}$ & & $99^{\mathrm{c}}$ \\
\hline 2 & & allyl bromide & r.t., $2 \mathrm{~h}$ & & $99^{c}$ \\
\hline 3 & & allyl bromide & r.t., $1 \mathrm{~h}$ & & $99^{c}$ \\
\hline 4 & & allyl bromide & r.t., $1 \mathrm{~h}$ & & $99^{\mathrm{c}}$ \\
\hline 5 & $\mathrm{~N}_{s}$ & allyl bromide & r.t., $1 \mathrm{~h}$ & & $99^{\mathrm{c}}$ \\
\hline 6 & (1) & propargyl bromide & r.t., $45 \mathrm{~min}$ & & $94^{\mathrm{c}}$ \\
\hline 7 & (2) & propargyl bromide & r.t., $2 \mathrm{~h}$ & & $99^{\mathrm{c}}$ \\
\hline 8 & (3) & propargyl bromide & r.t., $1 \mathrm{~h}$ & & $98^{\mathrm{c}}$ \\
\hline 9 & (4) & propargyl bromide & r.t., $1 \mathrm{~h}$ & & $99^{\mathrm{c}}$ \\
\hline 10 & (5) & propargyl bromide & r.t., $1 \mathrm{~h}$ & & $99^{\mathrm{c}}$ \\
\hline 11 & & propargyl bromide & $70{ }^{\circ} \mathrm{C}, 16 \mathrm{~h}$ & & $99^{\mathrm{c}}$ \\
\hline
\end{tabular}




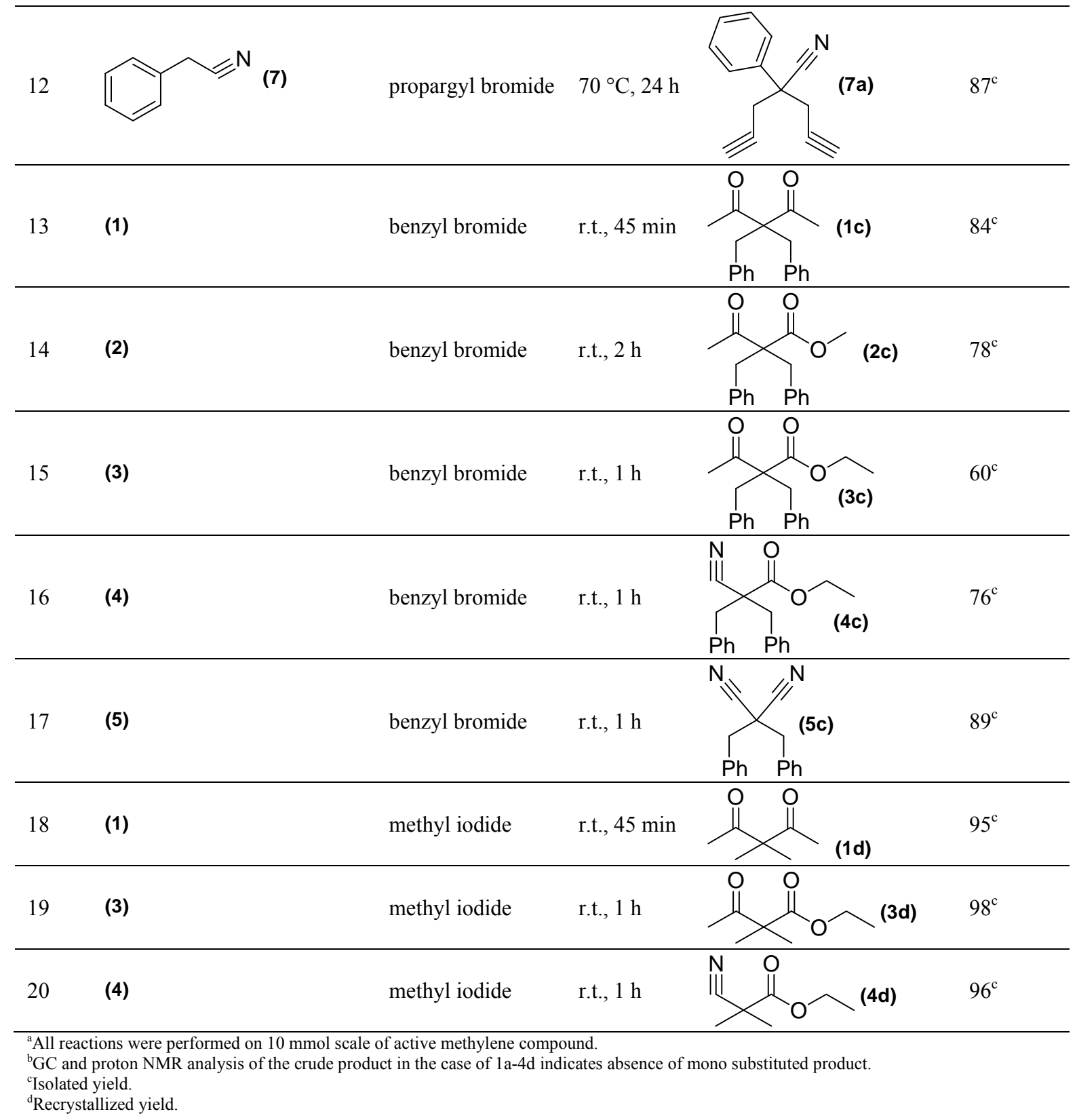

A comparison of the results of diallylation of acetyl acetone using present protocol and with various bases has been reported in Table 2 . As shown in Table 1 , a few other active methylene compounds $(\beta$ diketones, $\beta$-ketoesters and nitriles) also underwent a smooth alkylation with several reactive halides in the presence of cesium carbonate at room temperature, affording the corresponding di $C$-alkylated products in good to excellent yield and in good purity in a reasonably short period of time.

Table 2. Catalyst optimization for the synthesis of $3 \mathbf{a}$ under solvent- media

\begin{tabular}{llll}
\hline \multirow{2}{*}{ Entry } & Base & \multicolumn{1}{l}{ Yield ${ }^{\mathrm{a}, \mathrm{b}}(\%)$} & dialkylated \\
\cline { 3 - 4 } & & monoalkylated & 36 \\
\hline 1 & $\mathrm{NaOCH}_{3}$ & 39 & 61 \\
3 & $\mathrm{NaOC}_{2} \mathrm{H}_{5}$ & 34 & 46 \\
4 & $\mathrm{KOC}_{4} \mathrm{H}_{9}$ & 30 & 24 \\
5 & $\mathrm{NaH}$ & 25 & 75 \\
6 & $\mathrm{~K}_{2} \mathrm{CO}_{3}$ & 23 & 34 \\
7 & $\mathrm{~K}_{3} \mathrm{PO}_{4}$ & & 99 \\
\hline \multicolumn{2}{l}{${ }^{\mathrm{a}}$ All the reaction were performed on 10 mmol scale of active methylene compound for a duration of 45 min. }
\end{tabular}


As shown in Table 2, the dialkylation was fast in the case of cesium carbonate and proceeded to completion in 45 minutes itself compared to the reactions involving other bases. Further the reactions using other bases, led to a mixture of products, which could not be easily separated by normal purification methods. In the case of cesium carbonate, the reactions were clean and efficient. Importantly, in the present protocol the dialkylation reaction is free from any side product formation, such as monoalkylated product ${ }^{14-33}$, O-alkylation ${ }^{30}$ and condensation product. Bases like sodium ethoxide, potassium tert-butoxide, potassium phosphate and sodium hydride are known to give rise to considerable O-alkylation in DMF. We have observed only $C$-alkylation and very little, if at all, any O-alkylation.

Almost in all cases, the proton NMR spectrum and gas chromatogram of the crude product revealed its purity. The reactivity of the active methylene compounds towards the dialkylation under the present condition followed the anticipated trend, viz., $\beta$-diketone $>$ ethyl acetoacetate $=$ malononitrile $=$ ethyl cyanoacetate $>$ methyl acetoacetate $>$ diethyl malonate $>$ phenyl acetonitrile (see Table 1). Since cesium carbonate is known to be the base of choice for $N$-alkylation of amides ${ }^{7}$, it was of interest to study the alkylation of acetoacetanilide. Treatment of acetoacetanilide with alkyl halides in DMF in the presence of cesium carbonate at r.t., for $2 \mathrm{~h}$ resulted in complete consumption of acetoacetanilide and afforded the corresponding di $\mathrm{C}$-alkylated product without any $\mathrm{N}$-alkylation and other side products (Scheme 2).

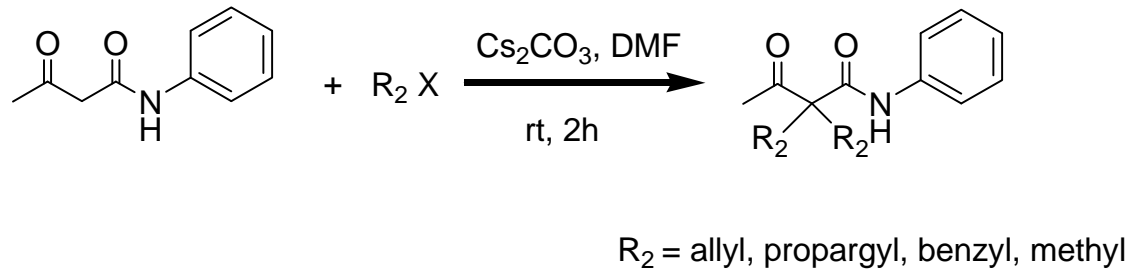

Scheme 2. Alkylation of active methylene compounds with amide functionality.

\section{Conclusion}

In summary we have found that dialkylation of active methylene compounds can be brought about efficiently, under mild conditions and under short duration, using cesium carbonate in DMF. No mono alkylated product was observed, hence the resulted products were attained with easy work up procedures.

\section{Acknowledgment}

Ulaganathan Sankar is thankful to Dr. K.K. Balasubramanian and the Prinicipal, Pachaiyappa's college (Affiliated to University of Madras) for providing the facilities for the work.

\section{Experimental Section}

\subsection{Materials and Methods}

All the reactions were carried out under nitrogen atmosphere, in all reaction $\mathrm{N}, \mathrm{N}^{\prime}-$ dimethylformamide was the choice of solvent. HPLC grade $\mathrm{N}, \mathrm{N}^{\prime}$ - dimethylformamide was used throughout all the reaction. Anhydrous cesium carbonates was purchased from commercial suppliers and was activated by heating at $180{ }^{\circ} \mathrm{C}$ for $2 \mathrm{hr}$ prior to use. All the other reagents were AR grade commercial materials and were directly used without further purification. Melting points were determined with Buchi melting B-545. TLC was carried out on a Merck silica gel $60 \mathrm{PF}_{254} \cdot{ }^{1} \mathrm{H},{ }^{13} \mathrm{C}$, Dept - NMR's was obtained from Bruker $(400 \mathrm{MHz} / 300 \mathrm{MHz})$ spectrometer in $\mathrm{CDCl}_{3}$. Low resolution mass spectra were recorded at ionizing voltage of eV by electron impact. Purity was recorded in Agilent-1200 series and Elemental analyses were recorded in vario micro super user. 


\subsection{General procedure}

To a solution of active methylene compound $(10 \mathrm{mmol})$ and cesium carbonate $(20 \mathrm{mmol})$ in DMF $(15 \mathrm{~mL})$ at $0{ }^{\circ} \mathrm{C}$, alkyl halide $(25 \mathrm{mmol})$ was added. The resulting mixture was stirred at r.t. (except Table 1 entry 11 and 12), under nitrogen atmosphere until reaction completion. The reaction was quenched by addition of water $(30 \mathrm{~mL})$ and then the mixture was extracted with MTBE $(3 \times 20 \mathrm{~mL})$, washed with water $(2 \times 20 \mathrm{~mL})$, saturated brine $(2 \times 20 \mathrm{~mL})$, and dried over $\mathrm{Na}_{2} \mathrm{SO}_{4}$. Evaporation of the solvent under vacuum furnished analytically pure dialkylated product (1a-4d). All the products exhibited satisfactory spectral data.

\subsection{Physical and Spectral Data}

3, 3-diallyl pentane-2, 4-dione (1a): Pale yellow liquid, yield: $99 \%{ }^{1} \mathrm{H}$ NMR (400 $\left.\mathrm{MHz}, \mathrm{CDCl}_{3}\right): \delta$ $2.03(6 \mathrm{H}, \mathrm{s}), \delta 2.57(4 \mathrm{H}, \mathrm{d}), 5.01(4 \mathrm{H}, 2 \mathrm{~d}), 5.40(2 \mathrm{H}, \mathrm{m}) .{ }^{13} \mathrm{C}$ NMR $\left(100 \mathrm{MHz}, \mathrm{CDCl}_{3}\right): \delta 26.6,34.5$, 69.7, 118.6, 131.5, 204.9. DEPT NMR (100 $\left.\mathrm{MHz} \mathrm{CDCl}_{3}\right): \delta 26.58,34.45,118.60,131.53$. GC purity: (99.62\%), Method: TRIAZWAX.M, RT- $9.5 \mathrm{~min}$. GCMS m/z $181(\mathrm{M}+\mathrm{H})^{+}, 165\left(\mathrm{M}^{+} \mathrm{CH}_{3}\right)^{+}$, $138\left(\mathrm{M}+\mathrm{H}-\mathrm{COCH}_{3}\right)^{+}, 123(\mathrm{M}-57)^{+}, 109(\mathrm{M}-71)^{+}, 97(\mathrm{M}-83)^{+}, 79(\mathrm{M}-101)^{+}$.

Methyl 2-acetyl-2-allyl pent-4-enoate (2a): Pale brown liquid, yield: 99.53\%. ${ }^{1} \mathrm{H}$ NMR (300 MHz, $\left.\mathrm{CDCl}_{3}\right): \delta 2.1(3 \mathrm{H}, \mathrm{s}), 2.53(4 \mathrm{H}, 2 \mathrm{~d}), 3.6(3 \mathrm{H}, \mathrm{s}), 5.06(4 \mathrm{H}, 2 \mathrm{~d}), 5.51(2 \mathrm{H}, \mathrm{m}) .{ }^{13} \mathrm{C} \mathrm{nmr}(75 \mathrm{MHz}$, $\left.\mathrm{CDCl}_{3}\right): \delta 26.9,35.9,52.3,63.3,119.1,132.1$. DEPT NMR $\left(100 \mathrm{MHz}, \mathrm{CDCl}_{3}\right): \delta 26.59,35.80$, 51.99, 118.79, 132.10. GC purity: (>96\%), Method: TRIAZDBW.M, RT-7.49 min. GCMS m/z 196

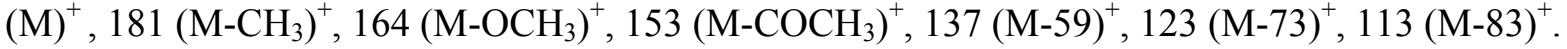

Ethyl 2-actyl-2-allylpent-4-enoate (3a): Pale yellow liquid, yield: 99.15\%. ${ }^{1} \mathrm{H}$ NMR (300 MHz, $\left.\mathrm{CDCl}_{3}\right): \delta 1.24(3 \mathrm{H}, \mathrm{t}), 2.13(3 \mathrm{H}, \mathrm{s}), 2.57(4 \mathrm{H}, 2 \mathrm{~d}), 4.16(2 \mathrm{H}, \mathrm{q}), 5.08(4 \mathrm{H}, 2 \mathrm{~d}), 5.5(2 \mathrm{H}, \mathrm{m}) .{ }^{13} \mathrm{C} \mathrm{NMR}$ $\left(100 \mathrm{MHz}, \mathrm{CDCl}_{3}\right): \delta 13.9,26.7,35.8,61.2,63.1,118.9,132.2,171.3,203.6$. DEPT NMR (100MHz, $\left.\mathrm{CDCl}_{3}\right): \delta 13.89,26.61,35.78,61.10,118.81,132.13$. GC purity: (>96\%), Method: TRIAZDBW.M,

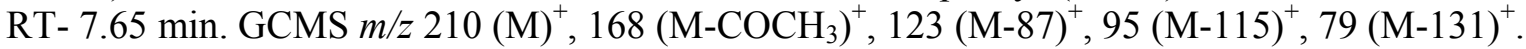

Ethyl 2-allyl-2-cyano pent-4-enoate (4a): Colorless liquid yield: 99\%, 1H NMR (300 MHz, $\left.\mathrm{CDCl}_{3}\right): \delta 1.27(3 \mathrm{H}, \mathrm{t}), 2.5(2 \mathrm{H}, \mathrm{d}), 2.6(2 \mathrm{H}, \mathrm{d}), 4.2(2 \mathrm{H}, \mathrm{q}), 5.2(4 \mathrm{H}, \mathrm{d}), 5.74(2 \mathrm{H}, \mathrm{m}) .{ }^{13} \mathrm{C} \mathrm{NMR}(100$ $\left.\mathrm{MHz}, \mathrm{CDCl}_{3}\right): \delta 13.9,40.5,49.2,62.5,118.2,120.7,130.5,167.7$. DEPT NMR $\left(100 \mathrm{MHz}, \mathrm{CDCl}_{3}\right): \delta$ 13.99, 40.49, 62.46, 120.7, 130.52. GC purity: (>99\%), Method: TRIAZDBW.M, RT- 7.96 min.

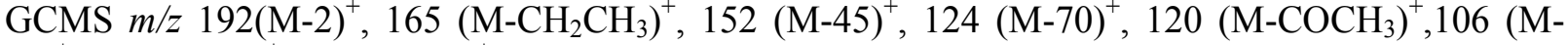
$88)^{+}, 93(\mathrm{M}-101)^{+}, 80(\mathrm{M}-114)^{+}$.

Diallylmalonate (5a): Pale brown liquid, yield 99\%, ${ }^{1} \mathrm{H}$ NMR (400 $\left.\mathrm{MHz}, \mathrm{CDCl}_{3}\right): \delta 2.68(4 \mathrm{H}, \mathrm{d})$, 5.39 (4H, 2d), $5.85(2 \mathrm{H}, \mathrm{m}) .{ }^{13} \mathrm{C}$ NMR $\left(100 \mathrm{MHz}, \mathrm{CDCl}_{3}\right): \delta 37.3,40.6,114.8,123.1,128.6$. DEPT NMR (100 MHz, CDCl3): $\delta 40.66,123.16,128.57$.GC purity: (>96\%), Method TRIAZDBW.M, RT11.2 min. GCMS m/z $146.18(\mathrm{M}-1)^{+}, 131(\mathrm{M}-15)^{+}, 119(\mathrm{M}-27)^{+}, 105(\mathrm{M}-41)^{+}, 68(\mathrm{M}-78)^{+}$.

3,3-diprop-2-ynyl pentane-2,4-dione (1b) : White solid, yield: 94\% [Compound was purified by tituration by using petroleum ether]. Melting range: $74.5-75.8{ }^{\circ} \mathrm{C} .{ }^{1} \mathrm{H}$ NMR $\left(400 \mathrm{MHz}, \mathrm{CDCl}_{3}\right): \delta$ $2.03(6 \mathrm{H}, \mathrm{s}), 2.19(6 \mathrm{H}, \mathrm{s}), 3.1(4 \mathrm{H}, \mathrm{s}) .{ }^{13} \mathrm{C} \mathrm{NMR}\left(100 \mathrm{MHz}, \mathrm{CDCl}_{3}\right): \delta 21.1,26.3,70,72.2,78.8$, 202.6. DEPT NMR $\left(100 \mathrm{MHz}, \mathrm{CDCl}_{3}\right): \delta 21.02,26.35,72.18,78.82$. GC purity: (>95\%), Method:

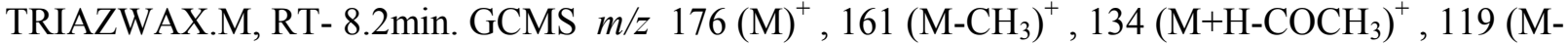
$57)^{+}, 95(\mathrm{M}-81)^{+}, 91(\mathrm{M}-85)^{+}$.

Methyl 2-acetyl-2-prop-2-ynylpent-4-ynoate (2b): Pale yellow solid, yield: 99\%, Melting range: 78.9- 81. $2^{\circ} \mathrm{C} .{ }^{1} \mathrm{H}$ NMR $\left(300 \mathrm{MHz}, \mathrm{CDCl}_{3}\right): \delta 2.02(2 \mathrm{H}, \mathrm{s}), 2.2(3 \mathrm{H}, \mathrm{s}), 2.88(4 \mathrm{H}, 2 \mathrm{~d}), 3.77(3 \mathrm{H}, \mathrm{s}) .{ }^{13} \mathrm{C}$ NMR $\left(75 \mathrm{MHz}, \mathrm{CDCl}_{3}\right): \delta 21.7,26,53.1,62.3,71.9,78.4,169.5,200.5$. DEPT-NMR $(100 \mathrm{MHz}$, $\left.\mathrm{CDCl}_{3}\right): \delta 21.76,26.08,53.13,72.02,78.41$. GC purity: (>97\%), Method: TRIAZDBW.M, RT- 9.27

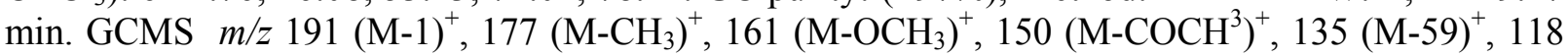


$(\mathrm{M}-74)^{+}, 111(\mathrm{M}-81)^{+}, 91(\mathrm{M}-101)^{+}, 79(\mathrm{M}-113)^{+}, 63(\mathrm{M}-129)^{+}$. Elemental analysis: Calculated for $\mathrm{C}_{11} \mathrm{H}_{12} \mathrm{O}_{3}$ : C, 68.74\%: H, 6.29\%: Found: C, 68.66\%: H, 6.26\%.

Ethyl 2-acetyl-2-prop-2-ynylpent-4-ynoate (3b): Pale brown liquid, yield: $98.5 \%$. ${ }^{1} \mathrm{H}$ NMR (400 $\left.\mathrm{MHz} \mathrm{CDCl}_{3}\right): \delta 1.26(3 \mathrm{H}, \mathrm{t}), 2.03(2 \mathrm{H}, \mathrm{s}), 2.2(3 \mathrm{H}, \mathrm{s}), 2.9(4 \mathrm{H}, 2 \mathrm{~d}), 4.22(2 \mathrm{H}, \mathrm{q}) .{ }^{13} \mathrm{C}$ NMR $(100$ $\left.\mathrm{MHz}, \mathrm{CDCl}_{3}\right): \delta 13.9,21.6,25.3,62.2,71.5,78.6,169.2,200.6$. DEPT NMR $\left(100 \mathrm{MHz}, \mathrm{CDCl}_{3}\right): \delta$ $13.79,21.53,25.79,62.04,72.02,78.38$. GC purity: (>97\%), Method-TRIAZDBW.M, RT- 9.39

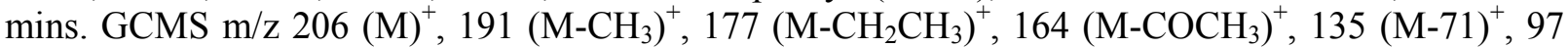
$(\mathrm{M}-99)^{+}$.

Ethyl 2-cyano-2-prop-2-ynylpent-4-ynoate (4b): Pale yellow liquid, yield: $99 \%{ }^{1} \mathrm{H}$ NMR (400 $\left.\mathrm{MHz}, \mathrm{CDCl}_{3}\right): \delta 1.32(3 \mathrm{H}, \mathrm{t}), 2.22(2 \mathrm{H}, \mathrm{s}), 2.92(4 \mathrm{H}, \mathrm{s}), 4.3(2 \mathrm{H}, \mathrm{q}) \cdot{ }^{13} \mathrm{C}$ NMR $\left(100 \mathrm{MHz}, \mathrm{CDCl}_{3}\right)$ : $\delta 13.9,25.7,47.3,63.6,73.7,76.2,117.1,165.9$. DEPT NMR $\left(100 \mathrm{MHz}, \mathrm{CDCl}_{3}\right): \delta 13.92,25.71$, 63.60, 73.71, 76.21. GC purity: (>99\%), Method: TRIAZDBW.M, RT- $10.36 \mathrm{~min}$. GCMS $\mathrm{m} / \mathrm{z}$ $190(\mathrm{M})^{+}, 160\left(\mathrm{M}-\mathrm{H}-\mathrm{CH}_{2} \mathrm{CH}_{3}\right)^{+}, 150(\mathrm{M}-40)^{+}, 144(\mathrm{M}-46)^{+}, 133(\mathrm{M}-57)^{+}, 122(\mathrm{M}-68)^{+}, 116(\mathrm{M}-74)^{+}$, $105(\mathrm{M}-85)^{+}, 89(\mathrm{M}-101)^{+}, 78(\mathrm{M}-112)^{+}$.

Diprop-2-yn-1-ylmalononitrile (5b): Pale brown solid, yield: 99\%, ${ }^{1} \mathrm{H}$ NMR $\left(400 \mathrm{MHz}, \mathrm{CDCl}_{3}\right): \delta$ $2.44(2 \mathrm{H}, \mathrm{s}), 3.09(4 \mathrm{H}, \mathrm{s}) .{ }^{13} \mathrm{C}$ NMR $\left(100 \mathrm{MHz} \mathrm{CDCl}_{3}\right): \delta 27.4,35.9,73.8,75.7,113.4$. DEPT NMR (100 MHz, CDCl3): $\delta 27.33,73.89,75.79$. GC purity: (>99\%), Method: TRIAZDBW.M, RT- 13.95 min. GCMS m/z $142.15(\mathrm{M}-1)^{+}, 115(\mathrm{M}-27)^{+}, 103(\mathrm{M}-39)^{+}, 88(\mathrm{M}-54)^{+}, 76(\mathrm{M}-66)^{+}$.

Diethyl diprop-2-ynyl malonate (6a): Pale yellow solid, yield 99\%, ${ }^{1} \mathrm{H}$ NMR (400 $\left.\mathrm{MHz}, \mathrm{CDCl}_{3}\right): \delta$ $\left.1.20(6 \mathrm{H}, \mathrm{t}), 2(2 \mathrm{H}, \mathrm{s}), 2.94(4 \mathrm{H}, \mathrm{s}), 4.16(4 \mathrm{H}, \mathrm{q}) .{ }^{13} \mathrm{C} \mathrm{NMR} 100 \mathrm{MHz}, \mathrm{CDCl}_{3}\right): \delta$ 13.8, 22.4, 56.2, 61.9, 71.6, 78.3, 168.4. DEPT NMR (100 MHz, $\left.\mathrm{CDCl}_{3}\right): \delta 13.87,22.37,61.85,71.68,78.30$. GC purity:

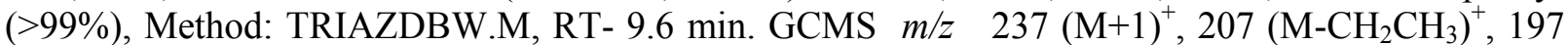
$(\mathrm{M}-39)^{+}, 191\left(\mathrm{M}-\mathrm{OCH}_{2} \mathrm{CH}_{3}\right)^{+}, 162\left(\mathrm{M}-\mathrm{COOCH}_{2} \mathrm{CH}_{3}\right)^{+}, 151(\mathrm{M}-85)^{+}, 133(\mathrm{M}-103)^{+}, 123(\mathrm{M}-113)^{+}$, $105(\mathrm{M}-131)^{+}, 91(\mathrm{M}-145)^{+}, 77(\mathrm{M}-157)^{+}, 65(\mathrm{M}-171)^{+}$.

2-phenyl-2-prop-2-ynyl pent-4-ynenitrile (7a): Pale yellow solid, yield: 87\%, ${ }^{1} \mathrm{H}$ NMR (400 MHz, $\left.\mathrm{CDCl}_{3}\right): \delta 2.17(2 \mathrm{H}, \mathrm{s}), 2.97(4 \mathrm{H}, 2 \mathrm{~d}), 7.27-7.55(5 \mathrm{H}, \mathrm{m}) .{ }^{13} \mathrm{C} \mathrm{NMR}\left(100 \mathrm{MHz}, \mathrm{CDCl}_{3}\right): \delta 29.1,45.6$, 73.1, 77.1, 120.4, 125.7, 128.4, 128.6, 135.9.DEPT NMR (100 MHz, $\left.\mathrm{CDCl}_{3}\right): \delta 29.11$ (down), 73.02, 77.23, 125.75, 128.38, 128.58. GC purity: (>98\%), Method: TRIAZDBW.M, RT- 13.50 min. GCMS $\mathrm{m} / \mathrm{z} 192(\mathrm{M}-\mathrm{H})^{+} 178(\mathrm{M}-15)^{+}, 165(\mathrm{M}-28)^{+}, 154(\mathrm{M}-39)^{+}, 127(\mathrm{M}-66)^{+}$.

3, 3-dibenzyl pentane-2, 4-dione (1c): White solid, yield 84\% [compound was purified by tituration by using petroleum ether]. ${ }^{1} \mathrm{H}$ NMR $\left(400 \mathrm{MHz}, \mathrm{CDCl}_{3}\right): \delta 2.14(6 \mathrm{H}, \mathrm{s}), 3.30(4 \mathrm{H}, \mathrm{s}), 7.03-7.28(10 \mathrm{H}$, m). ${ }^{13} \mathrm{C} \mathrm{NMR}\left(100 \mathrm{MHz}, \mathrm{CDCl}_{3}\right): \delta 28.4,37.5,72.1,126.9,128.5,129.8,136.1,206.74$. DEPT NMR $\left(100 \mathrm{MHz}, \mathrm{CDCl}_{3}\right): \delta 28.42,37.46,126.91,128.49,129.77$. HPLC purity: $(>99 \%)$, GCMS $\mathrm{m} / \mathrm{z}$ $280(\mathrm{M})^{+}, 237\left(\mathrm{M}-\mathrm{COCH}_{3}\right)^{+}, 189\left(\mathrm{M}-\mathrm{CH}_{2} \mathrm{Ph}\right)^{+}, 159(\mathrm{M}-121)^{+}, 147(\mathrm{M}-133)^{+}, 129(\mathrm{M}-151)^{+}, 91(\mathrm{M}-$ $189)^{+}$.

Methyl 2,2-dibenzyl-3-oxobutanoate (2c) : White solid, yield: 78\% [compound was purified by tituration by using petroleum ether]. ${ }^{1} \mathrm{H}$ NMR (400 MHz, $\left.\mathrm{CDCl}_{3}\right): \delta 1.96(3 \mathrm{H}, \mathrm{s}), 3.24(4 \mathrm{H}, \mathrm{s}), 3.69$ $(3 \mathrm{H}, \mathrm{s}), 7.13-7.31(10 \mathrm{H}, \mathrm{m}) .{ }^{13} \mathrm{C} \mathrm{NMR}\left(100 \mathrm{MHz}, \mathrm{CDCl}_{3}\right): \delta 28.7,39.4,51.6,65.7,126.5,127.9$, 129.6, 135.8, 171.7, 205.2. DEPT NMR $\left(100 \mathrm{MHz}, \mathrm{CDCl}_{3}\right): \delta 28.73,39.42,51.63,126.57,127.95$,

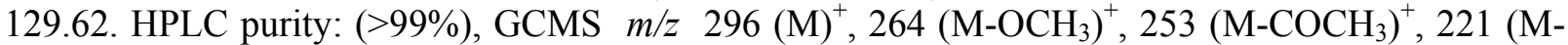
$59)^{+}, 205\left(\mathrm{M}-\mathrm{CH}_{2} \mathrm{Ph}\right)^{+}, 192(\mathrm{M}-104)^{+}, 173(\mathrm{M}-123)^{+}, 144(\mathrm{M}-152)^{+}, 131(\mathrm{M}-165)^{+}, 115(\mathrm{M}-181)^{+}, 103$ $(\mathrm{M}-193)^{+}, 91(\mathrm{M}-205)^{+}, 65(\mathrm{M}-231)^{+}$. Elemental analysis: calculated for $\mathrm{C}_{19} \mathrm{H}_{20} \mathrm{O}_{3}: \mathrm{C}, 77.00 \%: \mathrm{H}$, 6.80\%. Found: C, 77.06\%: H, 6.68\%:

Ethyl 2, 2-dibenzyl-3-oxobutanoate (3c): White solid, yield 60\% [compound was purified by tituration by using petroleum ether]. ${ }^{1} \mathrm{H}$ NMR $\left(400 \mathrm{MHz}, \mathrm{CDCl}_{3}\right): \delta 1.15(3 \mathrm{H}, \mathrm{t}), 1.64(3 \mathrm{H}, \mathrm{s}), 3.22$ $(4 \mathrm{H}, \mathrm{s}), 4.09(2 \mathrm{H}, \mathrm{q}), 7.12-7.28(10 \mathrm{H}, \mathrm{m}) .{ }^{13} \mathrm{C} \mathrm{NMR}\left(100 \mathrm{MHz}, \mathrm{CDCl}_{3}\right): \delta$ 13.8, 29.1, 39.8, 61.3, 66.2, 
126.9, 128.3, 130.1, 136.4, 171.7, 205.6. DEPT-NMR $\left(100 \mathrm{MHz}, \mathrm{CDCl}_{3}\right): \delta 13.87,29.10,39.86$,

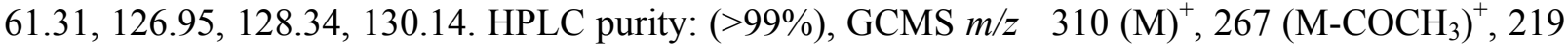
$\left(\mathrm{M}-\mathrm{CH}_{2} \mathrm{Ph}\right)^{+}, 173(\mathrm{M}-137)^{+}, 131(\mathrm{M}-179)^{+}, 91(\mathrm{M}-219)^{+}$.

Ethyl 2-benzyl-2-cyano-3-phenyl proponate (4c): Colorless oil, yield: 76\%, ${ }^{1} \mathrm{H}$ NMR (400 MHz, $\left.\mathrm{CDCl}_{3}\right): \delta 0.99(3 \mathrm{H}, \mathrm{t}), 3.13(2 \mathrm{H}, \mathrm{d}), 3.34(2 \mathrm{H}, \mathrm{d}), 4(2 \mathrm{H}, \mathrm{q}), 7.32-7.36(10 \mathrm{H}, \mathrm{m}) .{ }^{13} \mathrm{C} \mathrm{NMR}(100 \mathrm{MHz}$, $\left.\mathrm{CDCl}_{3}\right): \delta 13.7,43.4,53.3,62.6,118.6,127.9,128.6,130.1,134.1,168.2$. DEPT NMR (100 MHz, $\left.\mathrm{CDCl}_{3}\right): \delta 13.73,43.35,62.60,127.91,128.60,130.06$. HPLC purity: purity $(>99 \%), \quad \mathrm{GCMS} \mathrm{m} / \mathrm{z}$ : $293(\mathrm{M})^{+}, 202\left(\mathrm{M}-\mathrm{CH}_{2} \mathrm{Ph}\right)^{+}, 174(\mathrm{M}-119)^{+}, 91(\mathrm{M}-202)^{+}$.

Dibenzylmalononitrile (5c): White solid, yield: $89 \%$ [compound was purified by tituration by using petroleum ether]. ${ }^{1} \mathrm{H}$ NMR $\left(400 \mathrm{MHz}, \mathrm{CDCl}_{3}\right): \delta 3.27(4 \mathrm{H}, \mathrm{s}), 7.42(10 \mathrm{H}, \mathrm{m}) .{ }^{13} \mathrm{C} \mathrm{NMR}(100 \mathrm{MHz}$, $\left.\mathrm{CDCl}_{3}\right): \delta 41.2,43.4,115,128.8,129.1,130,132.1$. DEPT NMR $\left(100 \mathrm{MHz}, \mathrm{CDCl}_{3}\right): \delta 43.41$, $128.86,129.02,130.32$. HPLC purity: purity $(>99 \%)$, GCMS $m / z 246.3(\mathrm{M})^{+}, 91(\mathrm{M}-155)^{+}$.

3,3-dimethylpentane-2,4-dione (1d) : Yellow liquid, yield: 95\%, ${ }^{1} \mathrm{H}$ NMR $\left(400 \mathrm{MHz}, \mathrm{CDCl}_{3}\right): \delta$ $1.22(6 \mathrm{H}, \mathrm{s}), 2.01(6 \mathrm{H}, \mathrm{s}) .{ }^{13} \mathrm{C}$ NMR $\left(100 \mathrm{MHz}, \mathrm{CDCl}_{3}\right): \delta 20.9,25.8,62.2,207.5$. DEPT NMR $\left(100 \mathrm{MHz}, \mathrm{CDCl}_{3}\right): \delta 20.98,25.87$. GC purity: (94.85\%), Method: TRIAZHP5.M, RT -3.35 min. GCMS $m / z 129(\mathrm{M}=1)^{+}, 186\left(\mathrm{M}-\mathrm{COCH}_{3}\right)^{+}, 71(\mathrm{M}-59)^{+}$.

Ethyl 2,2-dimethyl-3-oxobutanoate (2d) : colorless liquid, yield: 98\%, ${ }^{1} \mathrm{H}$ NMR (400 $\mathrm{MHz}$, $\left.\left.\mathrm{CDCl}_{3}\right): \delta 1.24(3 \mathrm{H}, \mathrm{t}), 1.36(6 \mathrm{H}, \mathrm{s}), 2.16(3 \mathrm{H}, \mathrm{s}), 4(2 \mathrm{H}, \mathrm{q}) .{ }^{13} \mathrm{C} \mathrm{NMR} 100 \mathrm{MHz}, \mathrm{CDCl}_{3}\right): \delta$ 13.7, 21.5, 25.4, 26.7, 55.4, 61.1, 173.3, 205.4. DEPT NMR (100 MHz, $\mathrm{CDCl}_{3}$ ): $\delta 13.76,21.56$ (up), 25.39, 26.70, 61.01. GC purity: (>95\%), Method: TRIAZHP5.M, RT- $4.80 \mathrm{~min}$. GCMS m/z :159 (M+1) ${ }^{+}$, $116\left(\mathrm{M}-\mathrm{COCH}_{3}\right)^{+}, 88(\mathrm{M}-70)^{+}, 73(\mathrm{M}-85)^{+}$.

Ethyl 2-cyano-2-methyl propanoate (3d): Pale yellow liquid, yield: 96\%, ${ }^{1} \mathrm{H}$ NMR (400 MHz, $\left.\mathrm{CDCl}_{3}\right): \delta 1.33(3 \mathrm{H}, \mathrm{t}), 1.63(6 \mathrm{H}, \mathrm{s}), 4.25(2 \mathrm{H}, \mathrm{q}) .{ }^{13} \mathrm{C} \mathrm{NMR}\left(100 \mathrm{MHz}, \mathrm{CDCl}_{3}\right): \delta 13.7,24.5,38.4$, 62.5, 120.5, 169.4. DEPT-NMR (100 MHz, $\left.\mathrm{CDCl}_{3}\right): \delta 13.72,24.51,62.52$.GC purity:(>97\%),

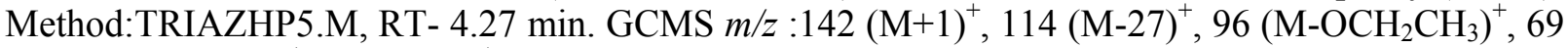
$\left(\mathrm{M}-\mathrm{COOCH}_{2} \mathrm{CH}_{3}\right)^{+}, 54(\mathrm{M}-87)^{+}$.

\section{References}

1. Wolfe, J. P., Buchwald, S. L. (1997) improved functional group compatibility in the palladiumcatalyzed amination of aryl bromides. Tetrahedron Lett. 38, 6359.

2. Cuny, G. D. (2003) Intramolecular ortho-arylation of phenols utilized in the synthesis of the aporphine alkaloids $( \pm)$-lirinidine and $( \pm)$-nuciferine. Tetrahedron Lett. 44, 8149.

3. (a) Parrish, J. P., Dueno, E. E., Kim, S., Jung, K. W. (2000) Improved $\mathrm{Cs}_{2} \mathrm{CO}_{3}$ promoted Oalkylation of acids. Synth. Commun., 30(15), 2687. (b) Dueno, E. E., Chu, F., Kim, S., Jung, K. W. (1999) "Cesium promoted O-alkylation of alcohols for the efficient ether synthesis" Tetrahedron Lett., 40, 1843.

4. Butler, J., Kellogg, R. M. (1981) Synthesis of sulfur-containing macrocycles using cesium thiolates. J. Org. Chem., 46, 4481.

5. Lavallée, J. F., Berthiaume, G., Deslongchamps, P., Grein, F. (1986) Intramolecular Michael addition of cyclic $\beta$-ketoester on conjugated acetylenic ketone. Tetrahedron Lett., 27, 5455.

6. Kumaraswamy, G., Pitchaiah, A., Ramakrishna, G., Ramakrishna, D. S., Sadaiah, K. (2006) Di$\mu$-hydroxy-bis $\left(N, N, N^{\prime}, N^{\prime} \text {-tetramethylenediamine)-copper(II)chloride[Cu(OH) } \mathrm{TMEDA}\right]_{2} \mathrm{Cl}_{2}$ : an efficient, practical catalyst for benzylation and allylation of amides. Tetrahedron Lett., 47, 2013.

7. Vogtle, F., Kissener, W. (1984) Cesium effect in C-C-bond forming reactions yielding large hydrocarbon rings. Chem. Ber.-Recueil, 117, 2538.

8. Dijkstra, G., Kruizinga, W. H., Kellogg, R. M. (1987) An assessment of the causes of the cesium effect. J. Org. Chem., 52, 4230.

9. Blum, Z. (1989) The cesium effect revisited, Acta Chemica Scandinavica, 43, 248. 
10. Ostrowicki, A., Koepp, E., Vogtle, F. (1992) The cesium effect - syntheses of mediocyclic and macrocyclic-compounds. Top. Curr. Chem., 161, 37.

11. Flessner, T., Doye, S. (1999) "Cesium carbonate: "a powerful inorganic base in organic synthesis," J. Prak. Chem., 341, 186.

12. Salvatore, R. N.: Nagle, A. S.: Jung, K. W. (2002) Cesium effect: high chemoselectivity in direct N-alkylation of amines. J. Org. Chem., 67, 674.

13. Suk, Y., Shibasaki, M. (1998) The asymmetric synthesis of cyclopentane derivatives by palladium-catalyzed coupling of prochiral alkylboron compounds .Tetrahedron Asymmetry, 9, 3751.

14. Vaughan, W. S., Gu, H. H., McDaniel, K. F. (1997) Reaction of tetracarbonyl ( $\pi$-allyl) manganese with carbon nucleophiles. Tetrahedron Lett., 38, 1885.

15. Laurenti, D., Feuerstein, M., Pepe, G., Doucet, H., Santelli, M. (2001) a new tetratertiary phosphine ligand and its use in Pd-catalyzed allylic substitution. J. Org. Chem., 66, 1633.

16. Kinoshita, H., Shinokubo, H., Oshima, k. (2004) water enables direct use of allyl alcohol for Tsuji-Trost reaction without activators. Org. Lett., 6, 4085.

17. King, J. F., Rathore, R., Lam, J. Y. L., Guo, Z. R., Klassen, D. F. (1992) PH optimization of nucleophilic reactions in water. J. Am. Chem. Soc., 114, 3028.

18. Yamamoto, Y., Nakagai, Y., Itoh, K. (2004) Ruthenium-catalyzed one-pot double allylation /cycloisomerization of 1,3-dicarbonyl compounds leading to exo-methylene cyclopentanes. Chem. Eur. J., 10, 231.

19. Kimura, M., Mukai, R., Tanigawa, N., Tanaka, S., Tamaru, Y. (2003) Triethylborane as an efficient promoter for palladium-catalyzed allylation of active methylene compounds with allyl alcohols. Tetrahedron, 39, 7767.

20. Curran, D. P., Seong, C. M. (1992) Radical annulation reactions of allyl iodomalononitriles. Tetrahedron, 48, 2175.

21. Diezbarra, E.: Delahoz, A., Moreno, A., Sanchezverdu, P. (1991) Phase transfer catalysis without solvent: selective mono- or di-alkylation of malononitrile. J. Chem. Soc. Perkin Trans., 1, 2589.

22. Muthusamy, S., Srinivasan, P. (2005) Facile chemoselective rhodium carbenoid of active methylene compounds. Tetrahedron Lett., 46, 635.

23. Reynolds, R. C., Trask, T. W., Sedwick, W. D. (1991) 2,4-Dichloro-5-(1-o-carboranylmethyl)-6methylpyrimidine: a potential synthon for 5-(1-o-carboranylmethyl)pyrimidines. J. Org. Chem., 56, 2391.

24. Bhar, S., Chaudhuri, S. K., Sahu, S.G., Panja, C. (2001) Solvent-free synthesis of 4,4-bisfunctionalized-1,6-dienes and 1,6-diynes on the surface of neutral alumina. Tetrahedron, 57, 9011.

25. Diez-Barra, E., De la Hoz, A., Moreno,A., Sanchez-Verdu, P. (1991) Phase transfer catalysis without solvent: selective mono- or di-alkylation of malononitrile. J. Chem. Soc. Perkin Trans., $1,2589$.

26. Shawn, R, H., Françoise, P., Van, A, M., Kim, F, A. (1990) Efficient synthesis of $\alpha-$ (hydroxymethyl) ketones not available through aldol-type processes. Synthesis, 11, 1059.

27. Marquet, J., Moreno-Manas, M., Pacheco, P., Prat, M., Katritzky. A. R., Brycki, B. (1990) Calkylation of $\beta$ diketones with benzylpyridinium salts. Evidence for chain radical mechanisms, Tetrahedron, 46, 5333.

28. Yoshihara, N., Kitagawa, T., Ihara, I., Hasegawa, S., Hasegawa, T. (1992) Selective $\alpha$-Monoand $\alpha, \alpha$-Dialkylations of 1,3-Diketones Using Solid Base Prepared from Sodium Ethoxide and Alumina. Bull. Chem. Soc. Jpn., 65, 1185.

29. Ridvan, L., Závada, J. (1997) Mono-vs.dialkylation of carbanions. Effects of absolute and relative acidity of the conjugate carbon acids in selectivity control. Tetrahedron, 53, 14793.

30. Ranu, B. C., Banerjee, S., Jana, R. (2007) Ionic liquid as catalyst and solvent: the remarkable effect of a basic ionic liquid, [bmIm] OH on Michael addition and alkylation of active methylene compounds. Tetrahedron, 63, 776. 
31. Rolin.y., Gebehenne.C., Derien. S., Dunach. E., Perichon. J. (1993) Electrochemical activation of zinc in the coupling reaction of $\alpha$-bromoesters with carbonyl compounds. J. Organomet. Chem., 461, 9.

32. Fotouhi, N., Joshi, P., Fry, D., Cook, C., Tilley, J. W., Kaplan, G., Hanglow, A., Rowan, K.., Schwinge, V., Wolitzky, B., (2000) The design and synthesis of potent cyclic peptide VCAMVLA-4 antagonists incorporating an achiral Asp-Pro mimetic. Bioorg. Med. Chem. Let, 10, 1171.

33. Baumstark, A. L., Vasquez, P. C., Chen, Y. X. (1994) Synthesis and thermolysis of ketal derivatives of 3-hydroxy-1, 2-dioxolanes . J. Org. Chem., 59, 6692. 